\title{
Applications of $\beta$-limit Dextrin as a Matrix Forming Excipient for Fast Disintegrating Buccal Dosage Formats
}

\author{
Xin $\mathrm{Qi}^{1}$, Richard Tester ${ }^{1}$, Yu Liu ${ }^{2}$ and Margaret Mullin ${ }^{3}$ \\ ${ }^{1}$ Glycologic Limited, 70 Cowcaddens Road, Glasgow, UK. \\ ${ }^{2}$ Department of Biological and Biomedical Sciences, Glasgow Caledonian University, Glasgow, UK. \\ ${ }^{3}$ School of Life Sciences, Glasgow University, Glasgow, UK.
}

Received, August 29, 2012; Revised, December 10, 2012; Accepted, December 23, 2012; Published, December 26, 2012.

\begin{abstract}
Purpose. To compare the properties of buccal delivery matrices (wafers) made with dextrin, $\beta$-limit dextrin and pre-gelatinised starch. Methods. The constituent $\alpha$-glucans were tested for their mucoadhesive properties in solution plus their content of crystalline material (differential scanning calorimetry, DSC). Wafers were made by lyophilisation of aqueous solutions/dispersions of the $\alpha$-glucans. Physical properties of the wafers were evaluated using texture analysis, dissolution coupled to photography and scanning electron microscopy (SEM). Results. The results highlighted how the $\beta$-limit dextrins chemical and physical properties were ideally suited for the production of buccal delivery wafers. Dissolution testing confirmed the excellent hydration profile of the $\beta$-limit dextrin (within wafers) with time. Using SEM it was evident that the homogeneous "bee-hive" like structure of the $\beta$-limit dextrin wafers, unlike the other $\alpha$-glucans, provided a rapidly hydratable strong porous matrix. Conclusions. The $\beta$-limit dextrin $\alpha$-glucan makes a superb (lyophilised) mucoadhesive delivery structure for the delivery of active agents to the buccal mucosa.
\end{abstract}

This article is open to POST-PUBLICATION REVIEW. Registered readers (see "For Readers") may comment by clicking on ABSTRACT on the issue's contents page.

\section{INTRODUCTION}

Oral fast disintegrating tablets, also known as 'fast-melt' or 'fast-dissolving' tablets (often referred to as 'wafers' by these authors), are solid single-unit dosage forms that dissolve or disintegrate rapidly in the oral cavity without the need for the administration of water (1). When the dosage forms come in contact with saliva, they disintegrate immediately and produce a suspension that can be easily swallowed by the patient (2). The format may, however, be used to deliver drugs into the mouth directly. This is often associated with bio-adhesion to the mucosa of the mouth. The active ingredients in the mouth are absorbed rapidly and move to the target organ/organs to produce the desired effect(s) (3). The oral delivery approach provides a better delivery system than conventional solid dosage forms which are swallowed (tablets and capsules) that can result in: compromised dosing accuracy; low drug availability at the site of action and; the necessity for frequent administration due to potentially extensive hepatic first-pass metabolism and pre-systemic drug degradation in the gastrointestinal (GI) tract (3). The oral dosage form is suitable for most patients including paediatric, geriatric, bed-ridden, nauseous or noncompliant patients. It is also preferred for people suffering from dysphagia, psychiatric patients, patients suffering from a variety of swallowing compromised disorders such as strokes, thyroid disorders, Parkinson's disease and other neurological disorders like multiple sclerosis and cerebral palsy (4). In addition to the ease of administration, the dosage format has also been found to improve the bioavailability of certain insoluble drugs (5). The physiology associated with buccal delivery is reviewed in detail elsewhere $(6,7)$.

The 'gold standard' at present for buccal delivery of drugs is based on gelatine. The Zydis format is well established (8). This protein provides an excellent structure and functionality in the mouth. The delivery matrices are made by lyophilisation and can be prepared with a range of different active pharmaceutical ingredients (APIs) and correspondingly for a number of conditions. However, as gelatine comes from an animal source it presents a number of issues - ethical, religious, health and functional that carbohydrates do not.

Corresponding Author: Xin Qi, Glycologic Limited, 70 Cowcaddens Road, Glasgow, UK; Email address: e.qi@glycologic.co.uk 
Hence, there are a number of commercial drivers to provide a plant derived material for this purpose. Mrsny (9) has reviewed recently approaches to drug delivery and readers are referred to this article for context. A more technically focused review has been published recently too (10) and readers are referred to this for a more physiological overview of oral delivery and systems developed for this purpose.

Different sugar and sugar alcohols can be formatted into fast dissolving tablets. Some are more compressible than others. Granulation has been used for this purpose where some of the sugars and sugar alcohols tested exhibited a disintegration time of less than ten seconds (11). However, these formats are not designed to provide buccal delivery with associated mucoadhesion.

In terms of the pharmaceutical sector, starch is one of the most widely used excipients. It is used for the manufacture of many solid dosage forms and can be formulated as a filler, disintegrant or binder (12). This may be in the native granular state or after one or more types of modification. Many starch based products are available on the market for these applications and are provided by a diverse array of companies.

Native starches (within species specific granule structures) consist of two types of $\alpha$ glucans: an essentially linear molecule called 'amylose' and a highly branched polymer called 'amylopectin'. Amylose molecules contain typically $>99 \% \alpha-(1-4)$ bonds with a very small proportion of $\alpha-(1-6)$ bonds. Amylopectin, however, contains around 95\% $\alpha-(1-4)$ and 5\% $\alpha$ (1-6) bonds. In general, native starches are insoluble but hydrate when in contact with water. Starches swell progressively as the water temperature is increased (eventually 'gelatinise') and have starch specific associated disintegration properties. As mentioned above, many starches are modified before use. The modification processes (which involve the alteration of physical and/or chemical characteristics) are designed to expand starch specific applications. This includes usage in pharmaceutical formulations which have been studied and utilised extensively over many years. The modifications are generally achieved through physical treatments (for example heat-moisture regimes etc.), enzymatic modification (e.g. amylase) and/or chemical derivatisation (addition of chemical groups).

In terms of physical modifications, pregelatinised starches have acquired a lot of interest with respect to the formulation of tablets, due to their cold water-swelling capacity and gel formation (13). They are produced by heating starches in water above their gelatinisation temperatures (where native crystalline structure is all or in part removed by the process) and then drying. This range of products (from different starches) are essentially amorphous and, when placed in water, have the advantage of dissolving or dispersing according to the concentration, even at room temperature (13). They are not, however, very stable in solution and retrograde (recrystallise) relatively quickly. A range of products are produced from different starches - especially maize.

Partial hydrolysis of starches yield dextrins (depolymerised starches), which have the same chemical arrangements as (their native) amylose and amylopectin molecules but with a lower degree of polymerisation. These molecules tend to be more soluble and stable in aqueous environments than the native starches they are formed from. Dextrins (discussed further below) contain some branching due to the presence of the $\alpha-(1-6)$ bonds. Usually dextrins comprise a polydisperse mix rather than a distinct single molecular species (14). Dextrins are produced typically by relatively dry heating (high temperature) of starches (usually with acid) although the term dextrin also includes products resulting from enzyme or acid-catalysed hydrolysis of wet starches (15). More extensive hydrolysis of starches with enzymes or acids generates glucose syrups.

Dextrins are used in different formats in pharmaceutical systems, and provide a number of technical advantages over other materials (16). As dextrins are readily degraded by $\alpha$-amylases to yield maltose and isomaltose (17) it has been suggested by many authors that they might be ideal for development as biologically compatible drug carriers (e.g. 18). Their structures do, however, present many technical limitations in pharmaceutical formulations.

The $\beta$-limit dextrins are derivatives of amylopectin (together with any branched amylose resides and maltose) representing 'trimmed' versions of starch $\alpha$-glucans (19). The dextrins should be produced by treating solubilised starch with pure $\beta$-amylase as the presence of $\alpha$-amylase will depolymerise the dextrin molecules. The $\beta$ amylase enzyme is an exo-enzyme that binds specifically to a maltose (or double ( $\alpha-(1-4)$-Dglucopyranosyl)) unit from the non-reducing ends of the $\alpha$-glucans and cleaves sequentially these maltose units off of the molecules. This process continues until the enzyme encounters any structural variation, producing maltose as sole hydrolysis product (plus the dextrin). In starch, 
amylopectins have been established for many decades to generate up to about $50 \%$ enzyme resistant material - $\beta$-limit-dextrins - bearing $\alpha-(1-$ 6)-D-glucose branched non-reducing ends (20), whereas non-branched amylose molecules are degraded completely to 'G-2' maltose (even chain lengths) or ' $\mathrm{G}-3$ ' maltotriose (for uneven chain lengths). Slightly branched amylose molecules are, like amylopectin, degraded from the non-reducing end of the molecules to the first branch points along the molecules. According to Bello-Pérez et al. (21) pure $\beta$-amylase acts 'only on the outer chains or on the outer parts of the inner chains of amylopectin'. These chains are depolymerised to stubs ultimately projecting from the branch points by the enzyme (22).

Because $\beta$-amylase cannot by-pass starch $\alpha$ (1-6) branches, the $\beta$-limit dextrin molecules contain all the original branches of the amylose or amylopectin molecules and exhibit the distribution of the internal chains of the original amylopectin macromolecule (22). The chain length distribution of the $\beta$-limit dextrins can be studied using, for example, high performance liquid chromatography (HPLC) after debranching with enzymes directed towards hydrolysing $\alpha-(1-$ 6 ) bonds, namely isoamylase and pullulanase. The specific hydrolysis profile of these dextrins represents the interior segments of the native molecules - between adjacent branch points (23). A peak chain length distribution degree of polymerisation (DP) of 40, 14 and 4 for debranched $\beta$-limit dextrin from waxy maize starch has, for example, been reported (24). Yuan et al. (25) reported that for waxy cereal starches there are a large molar proportion of short residual chains within $\beta$-limit dextrin, where about $70 \%$ of the total moles of residual chains occur between DP 3 and 16; with the modal value around DP 5. Similarly, in a study by Qi et al. (26) on maize starches, short chains with DP 14 and DP 5 represented about $90 \%$ of the population (on a weight basis).

The $\beta$-limit dextrins have a relatively high molecular weight - representing theoretically about half the molecular weights of the native amylopectin molecules. Molecular weights of 3.1 x $10^{6} \mathrm{D}$ have been reported for $\beta$-limit dextrins from waxy maize (27). A DP range (weight average) of $\sim 15,000-20,000$ (by size exclusion chromatography, SEC) has been obtained for $\beta$ limit dextrins produced from dull waxy maize starches (28) equivalent to 2.4 to $3.2 \times 10^{6} \mathrm{D}$. For wheat amylopectin, $\beta$-limit dextrin has been reported by Callaghan et al. (29) to have a molecular weight of $1.2 \times 10^{6} \mathrm{D}$.
Like other dextrins, $\beta$-limit dextrins are tasteless. If sugars are present, obviously they are slightly sweet. This does not provide any distinct technical advantage. However, uniquely they can be used as beverage clouding agents, thickeners (confectionery), carriers for volatile ingredients (e.g. for spray drying), bulking agents in low fat food products, fat replacers and to provide structure in extruded products $(28,30)$ due to their specific structure. In general terms, they are high molecular weight viscosity creating $\alpha$-glucans that can match the use of other dextrins in terms of calorie provision. However, the high molecular weight $\beta$-limit dextrins with their associated viscosity and solution properties (discussed in detail in a subsequent paper), are stable in solution due to their loss of external chains (by hydrolysis) - providing a unique solution matrix.

Although $\beta$-limit dextrins have been used for defining the specific structure of amylopectin (above), they have overall received little commercial interest in the food, clinical nutrition or pharmaceutical industries. This is due in part to commercial availability, lack of definition in terms of functional properties, registration (e.g. GRAS) status and associated costs of manufacture. As more applications are understood, however, the utilisation will increase.

The present study has been undertaken to present the possibilities of using $\beta$-limit dextrin as an excipient in fast disintegrating tablets ('wafers'). The intention is to present how the uniqueness of the dextrin structure and its associated properties in solution provide an excellent matrix for the purpose - rapid dissolution with associated drug delivery.

\section{MATERIAL AND METHODS}

\section{Materials}

The $\beta$-limit dextrin was prepared according to the method described by Qi and Tester (31). Dextrin (Crystal TEX 626) and pre-gelatinised starch (National B37) were obtained from National Starch and Chemical (Manchester, UK). Porcine mucin (M2378, type II, crude) and nutrient agar (70148) were purchased from Sigma (Gillingham, UK).

\section{Surface cohesion force measurement of $\alpha$ - glucans in solution}

The surface cohesion force of aqueous solutions of dextrin, $\beta$-limit dextrin or pre-gelatinised starch $(10 \%, w / v)$ were measured using a texture analyser (TA-XT2, Stable Micro Systems Ltd., Godalming, UK) with a compression platen probe (100 $\mathrm{mm}$ diameter). A petri-dish containing the 
test solution was fixed onto the platform of the texture analyser. The probe was lowered to come to contact with the surface of the liquid/gel and then was withdrawn at a rate of $5 \mathrm{~mm} \mathrm{~s}^{-1}$ for 20 $\mathrm{mm}$. The maximal force was recorded and used as the indicator of the cohesion (cohesive) force of the solution.

\section{Differential scanning calorimetry (DSC) of $\alpha$ - glucans}

The gelatinisation characteristics (temperatures and enthalpy) of dextrin, $\beta$-limit dextrin and pregelatinised starch were determined with a Mettler DSC 30 Low Temperature Cell controlled by a TC10A processor and TA 3000 thermal analysis system incorporating Mettler Toledo STARe software (32). Samples (3-3.5mg) were weighed accurately into standard $40 \mu \mathrm{l}$ aluminium pans to which 15 ul degassed deionised water was added and the contents were mixed. The pans were sealed with a ME 27330 press then the samples were heated from 5 to $100^{\circ} \mathrm{C}$ at $10^{\circ} \mathrm{C} \mathrm{min}^{-1}$ against pierced empty reference aluminium pan. The instrument was pre-calibrated using 3 metals (for temperature) and indium (for enthalpy) standards. All measurements were conducted in at least triplicate. After scanning, the samples were stored at $40^{\circ} \mathrm{C}$ for 17 days and rescanned in the DSC.

\section{Preparation of fast disintegrating wafers}

The wafers (oral delivery tablets) were prepared by dissolving/dispersing the required amount of dextrin, $\beta$-limit dextrin or pre-gelatinised starch into distilled water $\left(\sim 100 \mathrm{mg} \mathrm{ml}^{-1}\right)$ with vigorous stirring until no visible lumps/gel existed in the solution. Sample $(1 \mathrm{ml})$ aliquots of the aqueous solution were then transferred by pipette into small wells in blister (PVC/PVDC) packaging. The wafers were frozen at $-25^{\circ} \mathrm{C}$ before being lyophilised in a freeze - dryer (Supermodulyo $12 \mathrm{~K}$ from Edwards, Crawley, UK) at $-45^{\circ} \mathrm{C}$ and $10^{-1}$ mbar.

\section{Wafer properties}

Wafers were tested for thickness and weight variation to define any variability associated with the method of preparation. The thickness and diameter were determined using callipers (EHB, Germany) as the geometry of wafers may affect their disintegration time. Ten wafers were chosen randomly and the average value was determined. Uniformity and conformity of the wafers were determined by weighing ten wafers on a precalibrated analytical balance.

\section{Wafer strength testing}

Because of the lyophilised structure of the wafers, it was not possible to test the hardness of the wafers on a conventional hardness tester. Hence, the strength of the wafers was determined with a texture analyser (TA-XT2, Stable Micro Systems Ltd., Godalming, UK) as above. Individual wafers were placed on a support and deformed in a defined, controlled manner by a conical penetration probe over a constant distance of 1 mm using a speed of $0.1 \mathrm{~mm} \mathrm{~s}^{-1}$. A force $N$ versus - distance $(\mathrm{mm})$ profile was recorded. The maximal force $N$ after $1 \mathrm{~mm}$ of penetration was determined.

The results are presented as mean values $(n=6)$. The coefficient of variance $(\mathrm{CV})$ was less than $5 \%$ for the techniques used.

\section{Mucoadhesive properties}

Mucoadhesive properties of the wafers were also measured with a Texture Analyser (TA-XT2, Stable Micro Systems Ltd., Godalming, UK) as above using double strength nutrient agar (Fluka 70148) coated with an aqueous solution of porcine mucin $(0.5 \%$, Type II crude, M2378, Sigma) on the surface. The wafer was attached to the base of an aluminium compression platen probe $(37 \mathrm{~mm}$ diameter using double-sided adhesive tape) fixed to the mobile arm of the texture analyser. It was then lowered at a rate of $0.1 \mathrm{~mm} \mathrm{~s}^{-1}$ until contact with the nutrient agar was made. A contact force of $0.25 \mathrm{~N}$ was maintained for $60 \mathrm{~s}$, after which the probe was withdrawn at a rate of $1 \mathrm{~mm} \mathrm{~s}^{-1}$. The peak detachment force $(\mathrm{N})$ was recorded.

\section{Dissolution profile}

The dissolution profile of the wafers in water was monitored and photographed using a digital camera (Fujifilm S200EXR). The wafers were added carefully to a petri-dish containing $10 \mathrm{ml}$ distilled water at $25^{\circ} \mathrm{C}$ and the dissolution processes were recorded immediately and different times (s) thereafter.

\section{Scanning electron microscopy (SEM) of wafers}

Cross-section samples were prepared by cutting a thin slice of the wafers using a scalpel. The cut samples were mounted directly onto standard SEM specimen holders using double-sided copper adhesive tape (G3397, Agar Scientific, Stansted, UK). Since the wafers were electrically nonconductive, the samples were subjected to low vacuum gold/platinum sputter coating in the presence of argon gas (Polaron SC515, Fisons Instruments, Ipswich, UK). The samples were then examined and photographed using a JSM 
6400 Scanning Microscope (Jeol Ltd, Akishima, Japan) using an accelerating voltage of $3.0 \mathrm{kv}$ secondary electrons. Images were taken at $\mathrm{x} 30$, x100, x250 and x500 magnification and analysed by Scandium SEM image software.

\section{RESULTS}

\section{a-Glucans}

When a surface cohesive force is applied to the wafer making glucans (Figure 1) it is obvious how different the pre-gelatinised starch solution/gel responds to the pulling force compared to the $\beta$-limit dextrin and dextrin based solutions/gels. The peak force for the pregelatinised starch $(266.8 \mathrm{~g})$ is nearly five times that for $\beta$-limit dextrin (55.2 g) which is in itself nearly double that for dextrin $(36.1 \mathrm{~g})$. Hence, the ratio of the cohesive forces for the pre-gelatinised starch, $\beta$-limit dextrin and dextrin are 7.4:1.5:1.0.

\section{Differential scanning calorimetry (DSC)}

The DSC data (Figure 2) show that none of the (amorphous) glucans exhibited an amylopectin derived endotherm (a peak at about $65^{\circ} \mathrm{C}$ exists for most native starch amylopectin crystallites); since their semi-crystalline nature has been destroyed during processing. Any crystallinity (retrogradation) would thus have occurred during storage.

\section{Wafers}

Wafers made with the different $\alpha$-glucans were white and assumed the form of the freeze-drying blister packs. They all looked homogeneous although the pre-gelatinised starch wafers were less matt in colour than the dextrin wafers. The pre-gelatinised starch wafers appeared very dense.

The specific physical properties of the wafers are presented in Table 1 . The dimensions of the wafers $(1.85-1.88 \mathrm{~cm}$ in diameter and $0.34-0.38$ $\mathrm{cm}$ thick) were very similar. In terms of specific weights, the wafers were also similar - $92.0 \pm 1.9$, $97.2 \pm 1.3$ and $99.5 \pm 0.7 \mathrm{mg}$ for the dextrin, $\beta$-limit dextrin and pre-gelatinised starch respectively (probably reflecting the initial moisture content). The break strength of the wafers were however, very different and provided the most obvious differentiation - $1.9 \pm 0.6 \mathrm{~N}, 20.0 \pm 1.1 \mathrm{~N}$ and $53.7 \pm 1.1 \mathrm{~N}$ (or a ratio of $1.0: 10.5: 28.3$ ) for the dextrin, $\beta$-limit dextrin and pre-gelatinised starch wafers respectively. The dextrin wafers were fragile to the touch, the $\beta$-limit dextrin wafers were robust enough to be handled whilst the pregelatinised starch wafers were very hard.

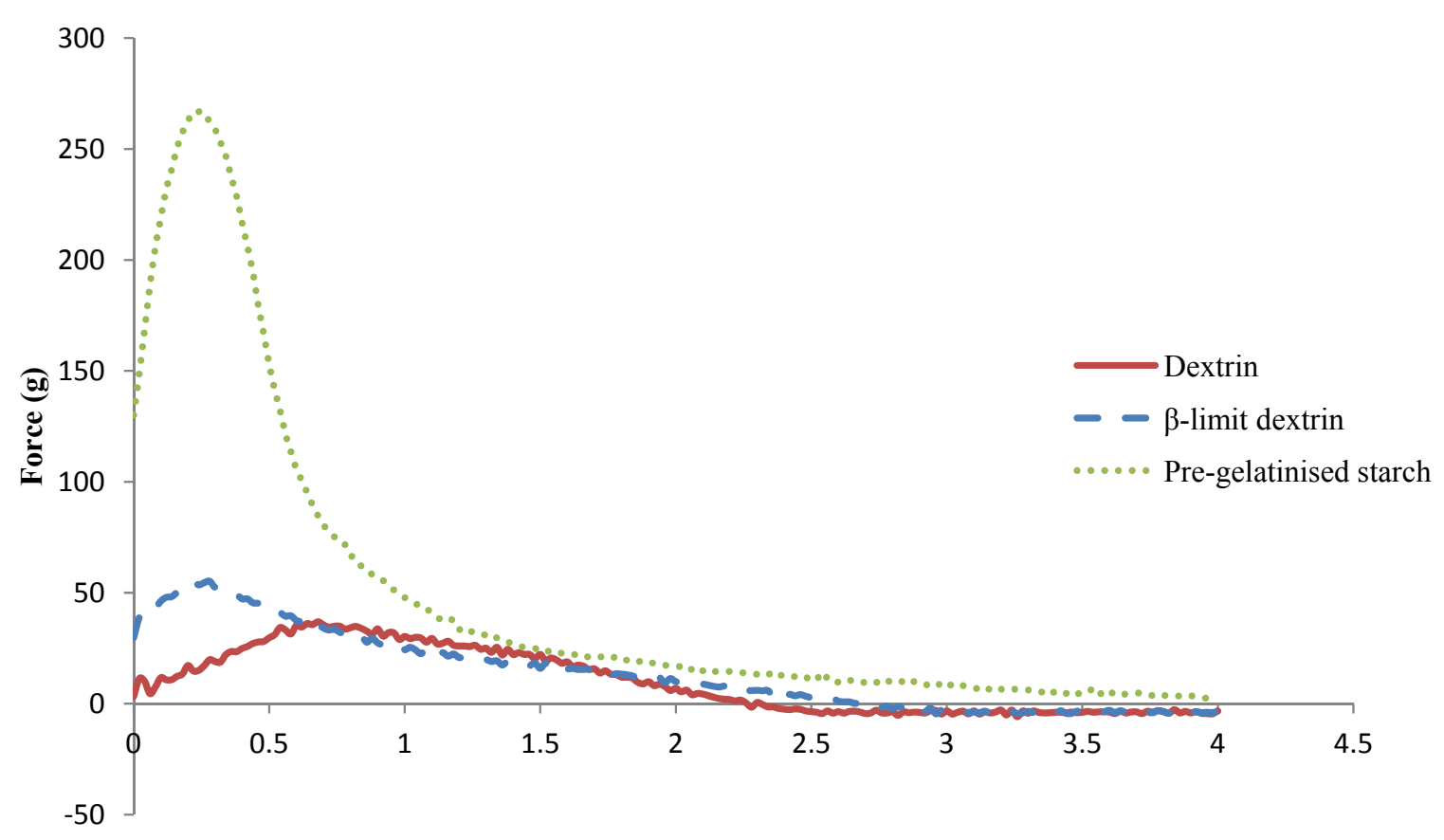

Time (s)

Figure 1. Comparison of surface cohesion force of aqueous solution/gel of dextrin, $\beta$-limit dextrin and pre-gelatinised starch $(10 \%, \mathrm{w} / \mathrm{v})$ 


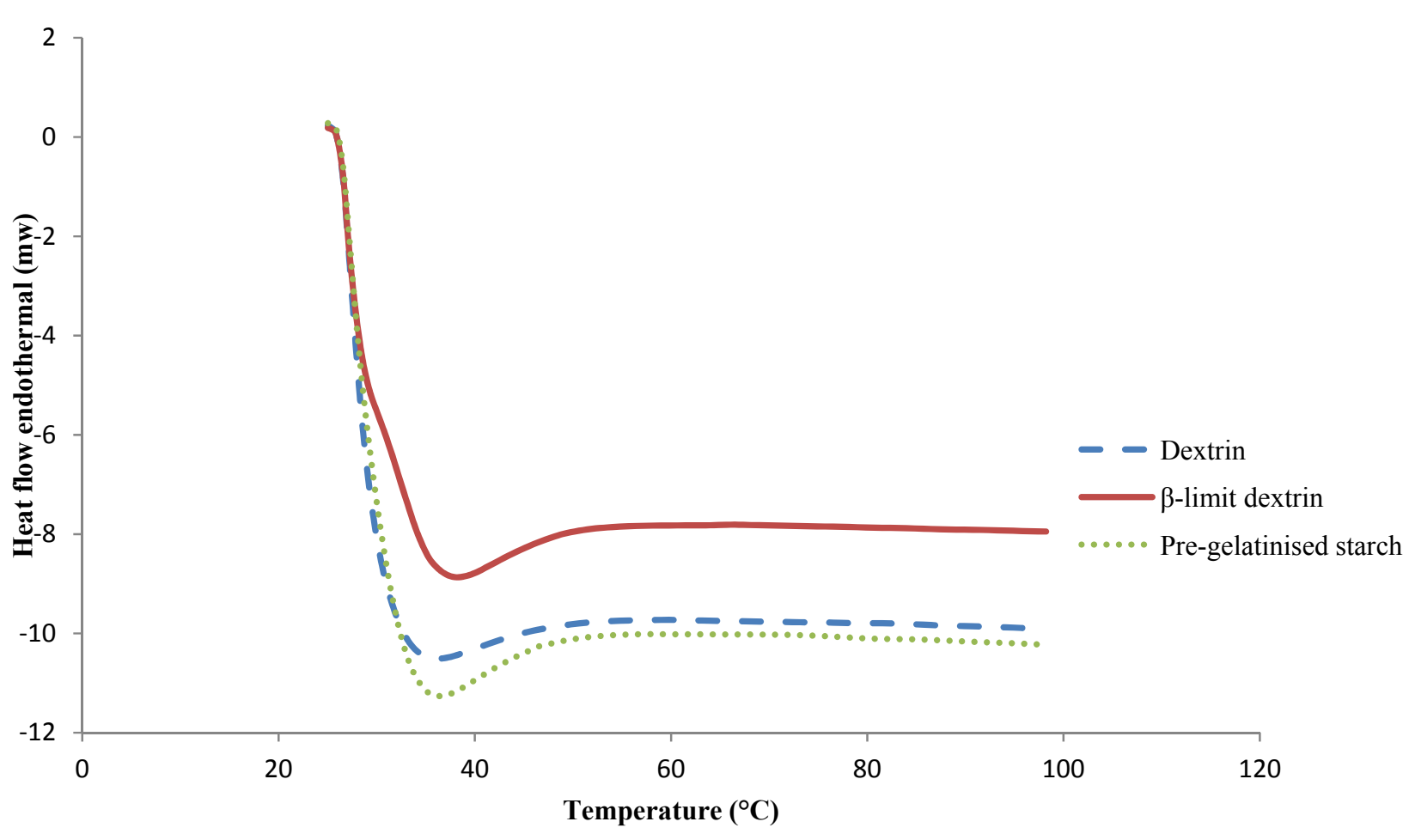

Figure 2. Comparison of DSC profiles of dextrin, $\beta$-limit dextrin and pre-gelatinised starch

Table 1. Technological characteristics of tablets

\begin{tabular}{lccc}
\hline & Dextrin & $\boldsymbol{\beta}$-limit dextrin & Pre-gelatinised starch \\
\hline Diameter (cm) & $1.88 \pm 0.03$ & $1.87 \pm 0.02$ & $1.85 \pm 0.02$ \\
Thickness (cm) & $0.34 \pm 0.02$ & $0.38 \pm 0.03$ & $0.37 \pm 0.02$ \\
Mean weight (mg) & $92.0 \pm 1.9$ & $97.2 \pm 1.3$ & $99.5 \pm 0.7$ \\
Strength (N) & $1.9 \pm 0.6$ & $20.0 \pm 1.1$ & $53.7 \pm 1.1$ \\
\hline
\end{tabular}

The $\beta$-limit dextrin wafers were far more mucoadhesive than the dextrin or pre-gelatinised starch wafers (Figure 3 ) in that the $\beta$-limit dextrin exerted about three times the force $(157.2 \mathrm{~g})$ of the dextrin $(64.9 \mathrm{~g})$ and pre-gelatinised starch $(53.7 \mathrm{~g})$. This is also reflected in an obvious difference by ratio: 2.9:1.5:1.0.

\section{Dissolution}

The dissolution properties of the wafers (in water at room temperature) are shown in Figure 4, for 0, 10,20 and $30 \mathrm{~s}$. It is apparent that the dextrin wafer disperses and dissolves very quickly $(<10 \mathrm{~s})$, the $\beta$-limit dextrin wafer remains intact after $10 \mathrm{~s}$, loses some structure by $20 \mathrm{~s}$ and has some residual structure at $30 \mathrm{~s}$. Unlike the dextrins, however the pre-gelatinised starch wafers remain intact during this time course (and did so for many hours thereafter).

\section{Appearance - SEM}

In terms of what makes the $\beta$-limit dextrin a 'better' carbohydrate with regard to its capacity to make wafers compared to the dextrin and pregelatinised starch, it is evident from the SEM images in Figure 5 that unlike the other two $\alpha$ glucans, the $\beta$-limit dextrin makes relatively homogenous honey-comb like structures, not randomised pockets of structure. The $\beta$-limit dextrin structure looks stronger intuitively due to the regularity and order. 


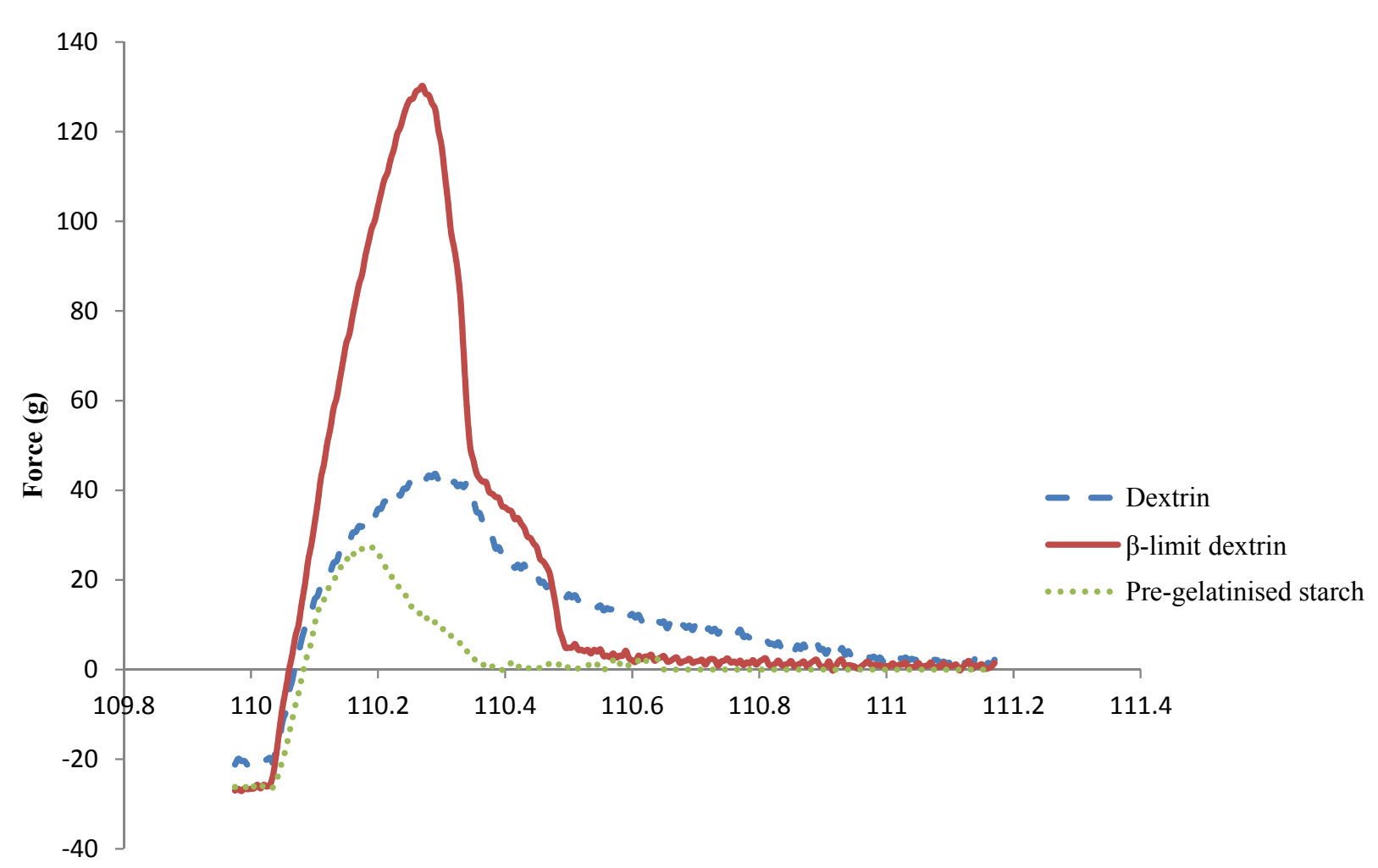

Time (s)

Figure 3. Comparison of mucoadhesive force between wafers made from dextrin, $\beta$-limit dextrin and pre-gelatinised starch

\section{DISCUSSION}

With respect to the $\alpha$-glucan properties in solution, pre-gelatinised starch molecules have a strong tendency to interact due to the thermodynamic favourability of amylose-amylose, amylopectinamylopectin and perhaps amylose-amylopectin molecular associations. These associations can then lead to strong retrograded (re-crystallised) structures which are hard to rupture. The long amylose molecules (especially) and the exterior amylopectin chains will associate as double helices and potentially crystalline regions containing ordered arrays of these helices depending on the processing conditions. Associations with other molecules may also occur with the $\alpha$-glucan chains in solution. These associations include fatty acids if present (forming for example 'amylose-lipid' complexes) although drug interactions may also occur (if present). The low molecular weight (linear) dextrins have the capacity to associate as helices and helical clusters like amylose and amylopectin. However, the shorter chain lengths, relatively high proportions of $\alpha-(1-6)$ bonds and special dissociation (in solution) make this less favourable. The relatively viscous solution of the $\beta$-limit dextrin system does not promote $\alpha$-glucan chain associations as the exterior linear chains of the amylopectin molecules have been removed. Thus they do not form helices easily or helical associations into crystallites. Their larger molecular size and longer internal chains (than dextrins) do, however, favour random physical molecular interactions and associations without the formation of helical structures.

The DSC data indicated that the $\alpha$-glucans were all amorphous and no residual semicrystalline structure impacted on the wafers. The pre-gelatinised starch (as discussed above) would be expected with time in solution, however, to form helical associations and probably contains some amylose single helical structures (e.g. amylose with lipid derived from the maize, dissociation temperatures $\sim 95-125^{\circ} \mathrm{C}$ ) and double helices (where higher temperature endotherms are created, $\sim 150-170^{\circ} \mathrm{C}$ ) generated during processing. When these associations form in aqueous environments the $\alpha$-glucans tend to precipitate (retrograde) from solution. For linear $\alpha$-glucans like amylose molecules this happens in fractions of seconds although for amylopectin this takes longer. The high temperature endotherms of maize amylose molecules have been presented 


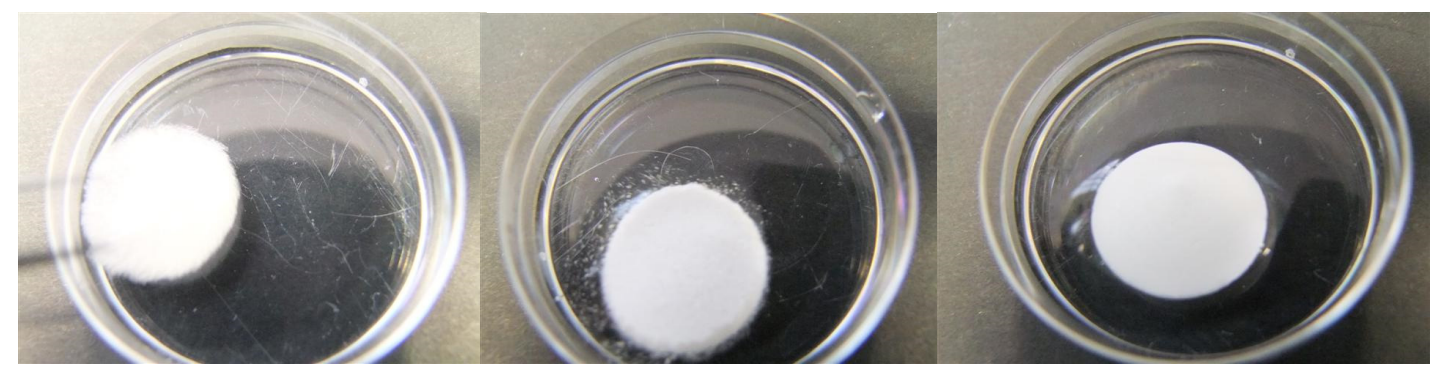

$0 \mathrm{~s}$

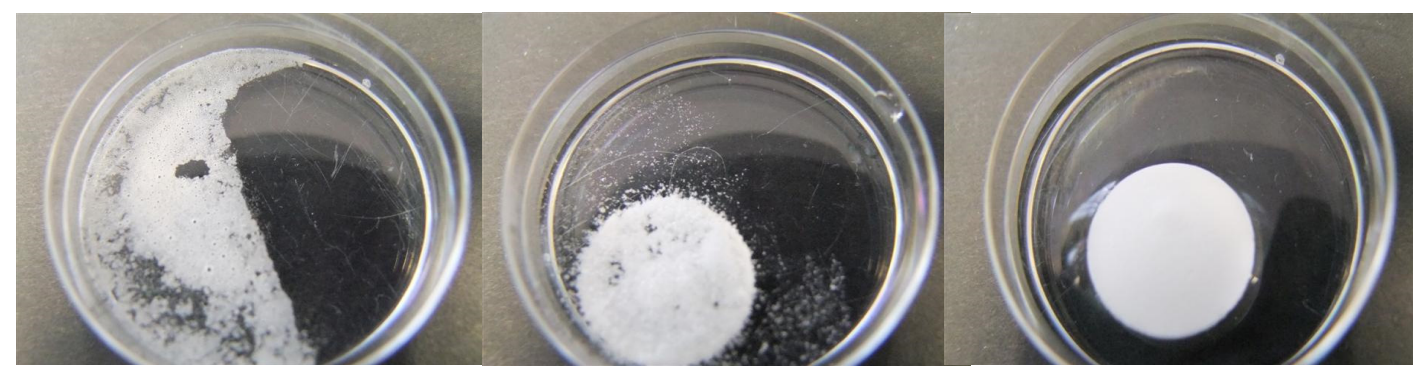

$10 \mathrm{~s}$

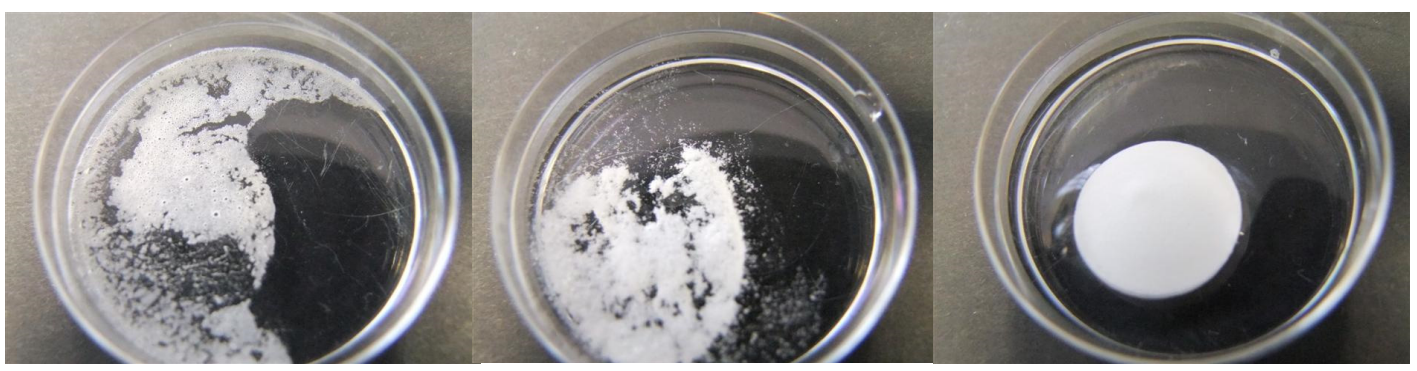

$20 \mathrm{~s}$

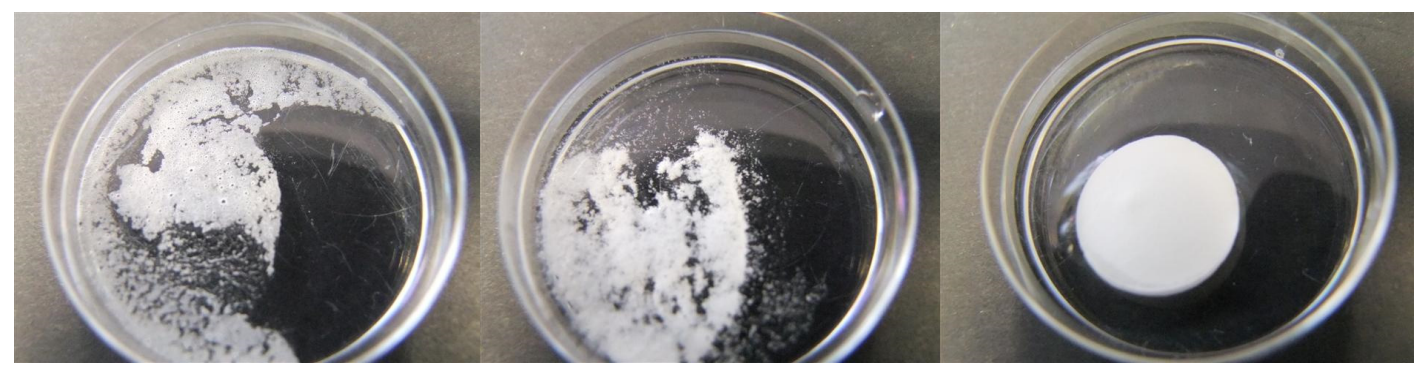

$30 \mathrm{~s}$

Dextrin wafer

$\beta$-limit dextrin wafer

Pre-gelatinised starch wafer

Figure 4. Dissolution profiles of dextrin, $\beta$-limit dextrin and pre-gelatinised starch wafers in water at $25^{\circ} \mathrm{C}$

and discussed elsewhere (e.g. 33). The formation of helices and crystallites before the formation of wafers or within wafers during drying would probably not be desirable as it would restrict hydration and dissolution. Since the exterior chains of $\beta$-limit dextrins have been removed by the $\beta$-amylase, these molecules will not associate as retrograded structures and then precipitate from solution. This is a very desirable attribute of the $\beta$-limit dextrins. The dextrins used in this study may retrograde with time where linear $\alpha$-glucans can associate if they exceed a degree of polymerisation (DP) of six. These could associate and form crystalline domains with time and 
retrograde from solution - similarly to regions of pre-gelatinised starch.

Retrogradation would lead to rigid hard to disperse structures. They may also appear gritty in the mouth. Hence, glassy/amorphous polymeric structures are essential for this purpose not crystalline structures.

It is difficult to imagine how the dextrin wafers could be packaged successfully without breaking during transit although this would appear not to be an issue for either the $\beta$-limit dextrin or pre-gelatinised starch. Clearly the high proportion of branches ( $\alpha-(1-6)$ bonds) in the $\beta$ limit dextrins with their high molecular weight provide a structure which can interact favourably with (the oral) mucosa. These data indicate why the $\beta$-limit dextrin is uniquely valuable for mucoadhesive applications.

Clearly there is a big difference in the visual hydration profiles of the wafers. These images (Figure 4) provide in vitro evidence for the beneficial role of the $\beta$-limit dextrin in oral delivery wafers. For many buccal delivery systems, a dissolution time of around twenty seconds is desirable with a retention of (some) structure to provide the base from which the drug is absorbed through the buccal mucosa.

In itself, fast dissolution is desirable for oral delivery. However, where drugs are intended to be absorbed thought the buccal mucosa it is also very desirable that they stick in a transient fashion to the inside of the cheeks (true buccal) or floor of the mouth (sublingual). The $\beta$-limit dextrin is heavily branched with hydroxyl groups projecting from all the glucose residues (as discussed above). These groups are very effective in making the wafers adhere to the buccal membranes while the structure makes an excellent mesh within which to carry and deliver the drugs. Other work (not presented here) has indicated no obvious interactions of the $\beta$-limit dextrins with drugs or other components of the wafers (flavours etc) to retard drug availability in vitro or in vivo. This is important to ensure that rapid transfer of any active can occur across the buccal membranes and into the circulatory system.

Salivary amylase acts on the $\alpha-(1-4)$ bands of the $\beta$-limit dextrin generating lower molecular weight dextrins plus some sugars (maltose and glucose). This causes the delivery system to be removed from the mouth post drug delivery leaving a fresh and clean mouth feel. Because non-starch polysaccharides cannot be hydrolysed, they remain in the mouth and provide a very unpleasant sensation when struck to the teeth, gums etc. Hence, the dissolution profiles (Figure 4) does not reveal the full physiological value of the wafers - but does show the rapid nature of the dissolution.

In the buccal cavity there would be far less water with the presence of the oral mucosa to adhere to. Hence, the system employed here (Figure 4) is far more extreme than that experienced in the mouth - where mucoadhesion also occurs. However, it does provide an excellent medium with which the dissolution can be followed photographically.

Zydis type (lyophilised) gelatine wafers dissolve 'instantaneously' in the mouth and can be used to deliver drugs through the oral mucosa or as a consequence of swallowing (8). Bioequivalence studies have been made between this system and other delivery formats. Different sugar and sugar alcohols can be used in combination with the gelatine in lyophilised wafers, where typical disintegration times of between around about ten to thirty seconds can be achieved (1). These data are comparable to the data reported in this study for the $\beta$-limit dextrin.

Clearly the $\beta$-limit dextrin structure (as visualised during this study) optimises strength whilst also providing capillaries for rapid hydration. This is quite unexpected but does provide an intriguing insight into the nature of materials suitable for this purpose.

It is recognised that with all new excipients one of the key challenges (apart from supply and cost) is GRAS acceptance. However, $\beta$-limit dextrin is a GRAS permitted dextrin which facilitates commercial uptake and utilisation in the pharmaceutical (and food) sectors (34).

Images (SEM) of gelatine based oral delivery structures (wafers) have been presented by for example Chandrasekhar et al. (1). These structures do look similar to the $\alpha$-glucan images presented here (Figure 5) - possibly most like the $\beta$-limit dextrin. This is perhaps not surprising in view of the processing regimes used but perhaps more so in that gelatine is present in the Chandrasekhar et al. (1) formulations. When Chandrasekhar et al. (1) used mannitol or viscosity modifying polymers, however, in their formulations they looked different.

Overall, these data indicate that lyophilised $\beta$ limit dextrin based matrices provide an attractive opportunity for oral drug delivery with many advantages over gelatine. Future papers discuss specific applications of this technology with specific drugs. 

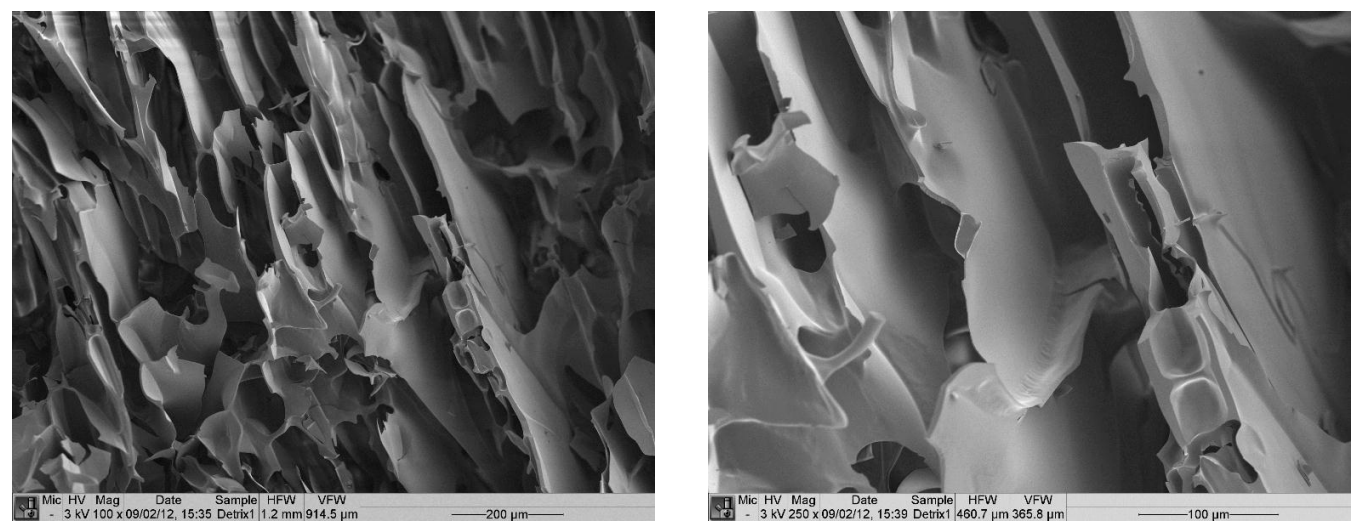

Dextrin
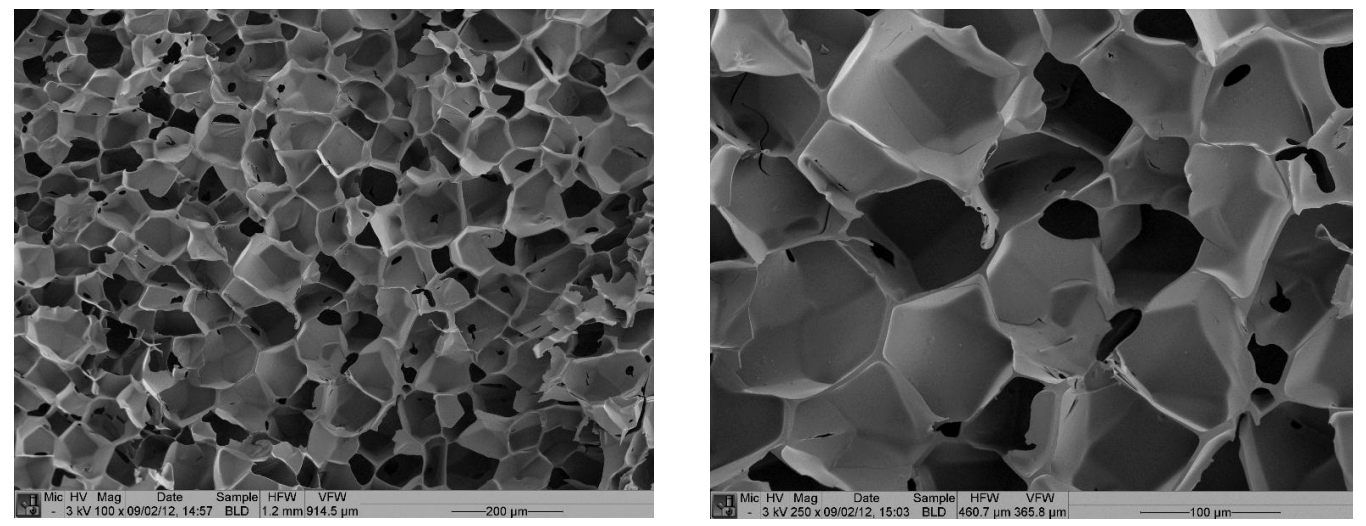

$\beta$-limit Dextrin
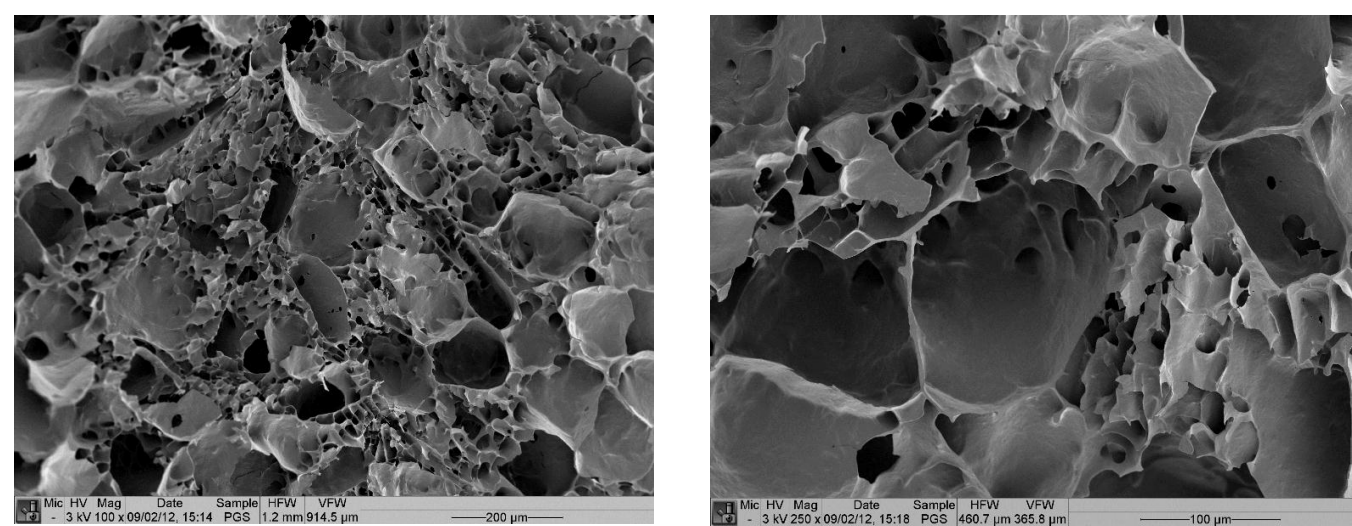

Pre-gelatinised starch

Figure 5. Scanning electron microscopy (SEM) images of wafers made from dextrin, $\beta$-limit dextrin and pregelatinised starch.

\section{CONCLUSIONS}

The less commonly studied dextrin generated by the action of $\beta$-amylase on amylopectin - $\beta$-limit dextrin - creates excellent oral delivery matrices when lyophilised. The properties of these 'wafers' make them highly desirable for buccal delivery.
The combination of strength and functionality provide many opportunities for utilisation to deliver active ingredients - includes drugs - to the oral cavity. 


\section{REFERENCE}

1. Chandrasekhar R, Hassan Z, AlHusban F, Smith AM, Mohammed AR. The role of formulation excipients in the development of lyophilised fast disintegrating tablets. Eur J Pharm Biopharm, 2009; 72:119-129.

2. Sastry SV, Nyshadham JR, Fix JA. Recent technological advances in oral drug delivery - a review. Pharm Sci Tech Today, 2000; 3:138-145.

3. Virely P, Yarwood R. Zydis - a novel fast dissolving dosage form. Manuf Chem, 1990; 61:36-37.

4. Badgujar BP, Mundada AS. The technologies used for developing orally disintegrating tablets: A review Acta Pharm, 2011; 61:117-139.

5. Ahmed IS, Nafadi MM, Fatahalla FA. Formulation of fast-dissolving ketoprofen tablet using freezedrying in blisters technique. Drug Dev Ind Pharm, 2006; 32:437-442.

6. Hoogstraate JAJ, Wertz PW. Drug delivery via the buccal mucosa. PSTT, 1998; 1(7):309-316.

7. Salamat-Miller N, Chittchang M, Johnston TP. The use of mucoadhesive polymers in buccal drug delivery. Adv Drug Del Rev, 2005; 57:1666-1691.

8. Seager H. Drug-delivery products and the Zydis fast dissolving dosage form. J Pharm Pharmacol, 1998; 50:375-382.

9. Mrsny RJ. Oral drug delivery research in Europe. J Control Release, 2012; 161:247-253.

10. Hearden V, Sankar V, Hull K, Juras DV, Greenberg M, Kerr AR, Lockhart PB, Patton LL, Porter S, Thornhill MH. New developments and opportunities in oral mucosal drug delivery for local and systemic disease. Adv Drug Del Rev, 2012; 64:16-28.

11. Mizumoto T, Masuda Y, Yamamoto T, Yonemochi E, Terada K. Formulation design of a novel fastdisintegrating tablet. Int J Pharm, 2005; 306:83-90.

12. Visavarungroj N, Remon J. Evaluation of maltodextrin as binding agent. Drug Dev Ind Pharm, 1992; 18 (15): 1691-1700.

13. Pifferi G, Santoro P, Pedrani M. Quality and functionality of excipients. IL Farmaco, 1999; 54:114.

14. Tester RF. $\beta$-Limit dextrin - a new food and pharmaceutical resource. Starch/Stàrke, 2005; 57:442-451

15. Ohkuma, K.; Wakabayashi, S., Fibersol-2: Soluble, Non-Digestible, Starch-Derived Dietary Fibre. Advanced Dietary Fibre Technology, Blackwell Science Ltd., London, pp 509-522, 2001.

16. Alvani K, Qi X, Tester RF. Use of carbohydrates, including dextrins, for oral delivery. Starch/Stàrke, 2011; 63:424-431.

17. Davies DS. Kinetics of icodextrin. Perit Dial Int, 1994; 14:S45-S50.

18. Hreczuk-Hirst D, Chicco D, German L, Duncan R. Dextrins as potential carriers for drug targeting: Tailored rates of dextrin degradation by introduction of pendant groups. Int J Pharm, 2001; 230:57-66.
19. Yun SH, Matheson NK. Structures of the amylopectins of waxy, normal, amylose-extender, and wx-ae genotypes and of the phytoglycogen of maize. Carbohydr Res, 1993; 243:307-321.

20. Whelan WJ. Hydrolysis with $\beta$-amylase and preparation of the $\beta$-amylase limit dextrin of amylopectin. Methods Carbohydr Chem, 1964; 4:261.

21. Bello-Pérez LA, Paredes-López O, Roger P, Colonna $\mathrm{P}$. Molecular characterization of some amylopectins. Cereal Chem, 1996; 73 (1):12-17.

22. Tester RF, Qi X. $\beta$-Limit dextrin - properties and applications. Food Hydrocolloid, 2011; 25:18991903.

23. Thompson DB. On the non-random nature of amylopectin branching. Carbohyd Polym, 2000; 43:223-239.

24. Akai H, Yokobayashi K, Misaki A, Harada T. Structural analysis of amylopectin using pseudomonas isoamylase. Biochimica et Biophysica Acta (BBA). 1971; 252(3):427-431.

25. Yuan RC, Thompson DB, Boyer CD. Fine structure of amylopectin in relation to gelatinization and retrogradation behaviour of maize starches from three wx-containing genotypes in two inbred lines. Cereal Chem, 1993; 70:81-89.

26. Qi X, Tester RF, Snape CE, Ansell R. The effect of annealing on the structure and gelatinization of maize starches with amylase dosage series. Prog Food Biopolym Res, 2005; 1:1-27.

27. Tester RF, Qi X. A chemical carrier based on a betalimit dextrin. WO2004014156, 2004.

28. Ammeraal R, Friedman R. Beta-limit dextrin from dull waxy starches. US patent 005482560 , American Maize Technology, Inc., Dimmitt, Tex, 1996.

29. Callaghan PT, Lelievre J, Lewis JA. A comparison of the size and shape of $\beta$-limit dextrin and amylopectin using pulsed field-gradient nuclear magnetic resonance and analytical ultracentrifugation. Carbohydr Res, 1987; 162:33-40.

30. Cho, S.; Prosky, L.; Dreher, M. L., Complex Carbohydrates in Foods. Marcel Dekker, USA, pp 431-593, 1999.

31. Qi X, Tester RF. Bioadhesive Properties of $\beta$-limit dextrin. J Pharm Pharmaceut Sci, 2011; 14 (1):60-66.

32. Tester RF, Morrison WR. Swelling and Gelatinization of Cereal Starches. I. Effects of Amylopectin, Amylose, and Lipids. Cereal Chem, 1990; 67:551-557.

33. Chung H-J, Jeong H-Y, Lim S-T. Effects of acid hydrolysis and defatting on crystallinity and pasting properties of freeze-thawed high amylose corn starch. Carbohyd Polym, 2003; 54:449-455.

34. Food additives permitted for direct addition to food for human consumption. Subpart I-Multipurpose additives. Sec. 172.892 Food starch-modified. FDA part 172. 2012. 